\title{
Anti-diabetic Properties of Thymoquinone is unassociated with Glycogen Phosphorylase Inhibition
}

\author{
Nahid Mahmoud Hassan El-Ameen ${ }^{1 *}$, Manal Mohamed Elhassan Taha ${ }^{1 *}$, Siddig Ibrahim Abdelwahab', Asaad Khalid', Fatima Elfatih' ${ }^{2}$, \\ Mona Awad Kamel' ${ }^{1}$ and Bassem Yousif Sheikh ${ }^{3}$
}

'Department of Biomedical Research Unit, Researcher at Medical Research Center, Jazan University, Jazan, Saudi Arabia.

2Department of Biochemistry Medicinal and Aromatic Plants Research Institute, National Centre for Research, P. O. Box 2420 Khartoum, Sudan.

${ }^{3}$ Department of Surgery, MABL Chair, College of Medicine, Taibah University, Saudi Arabia.

\begin{abstract}
Background: Nigella sativa L. (Black seed), is commonly used by traditional healers as a remedy for more than four thousand years. The antidiabetic property of $N$. sativa seeds oil is attributable to the presence of Thymoquinone (TQ). On the other hand many studies have been designed to investigate the possible effects of the TQ in Streptozotocin (STZ) and nicotinamide (NA)-induced diabetes in rats. Aim of the study: The aim of this study was to elucidate the mechanisms underlying the glucose lowering effects of thymoquinone. Methods: In vitro and in silico using glycogen phosphorylase (GPa) enzyme assay and docking tools were used. Results: Oral administration of TQ for 60 days, dose dependently improved the glycemic status in STZ-NA induced diabetic rats. GPa activity was measured in the direction of glycogen synthesis by the release of phosphate from glucose-1-phosphate. TQ at a concentration of $0.05 \mathrm{Mm}$ inhibits GPa activity by only $14.9 \%$. Conclusion: These results show that $\mathrm{TQ}$ at $60 \mathrm{mg} / \mathrm{kg} \mathrm{b.w}$ is associated with potential antihyperglycemic effects. Furthermore, anti-diabetic properties of TQ are unassociated with glycogen phosphorylase inhibition.
\end{abstract}

Key words: Diabetes, Docking, Enzyme, Glycogen phosphorylase inhibition, Streptozotocin, Thymoquinone.

\section{SUMMARY}

- Oral administration of Thymoquinone for 60 days, dose dependently improved the glycemic status in STZ-NA induced diabetic rats.

- Thymoquinone at a concentration of $0.05 \mathrm{Mm}$ inhibits GPa activity by only $14.9 \%$.

- Anti-diabetic properties of TQ are unassociated with glycogen phosphorylase inhibition.

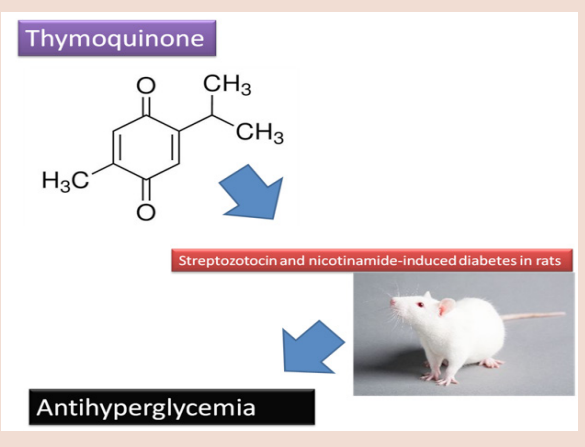

PICTORIAL ABSTRACT

Abbreviations used:TQ: Thymoquinone, STZ: Streptozotocin, NA: Nicotinamide, GTT: Glucose tolerance test, ADT: Auto Dock Tools, GPa: Glycogen phosphorylase, GFP: Grid parameter file, PDB: Protein data bank.

Correspondence:

Dr. Manal Mohamed ElhassanTaha, Department of Biomedical Research Unit, Researcher at Medical Research Center, Jazan University, Jazan, Saudi Arabia. Phone no: 00966506653828

Email: manalroa@yahoo.com DOI : 10.5530/pj.2015.6.16

\section{INTRODUCTION}

Nigella sativa L. (Black seed), is commonly used by traditional healers as a remedy for more than four thousand years. ${ }^{1,2}$ It has been used to treat many ailments including urine retention, hyperglycemia, cancer, pain, inflammation and diabetes. ${ }^{3-6}$ Phytochemicals with versatile structures (contains alkaloids, tannins, steroids and flavonoids) were isolated from black seeds. ${ }^{2,7,8}$ The antidiabetic property of $N$. sativa seeds oil is attributable to the presence of Thymoquinone (TQ). TQ is the main ingredients of black seeds oil and has been reported to have various pharmacological activities. On the other hand many studies have been designed to investigate the possible effects of the TQ in streptozotocine (STZ)-induced diabetes in rats. Previous findings demonstrated that the anti-diabetic action of TQ is mediated through a decrease in hepatic gluconeogenesis or by modifying the levels of glycoprotein components in plasma and tissues of diabetic rats. TQ was also found to exert curative protective properties in diabetes by diminishing oxidative stress and conserving pancreatic $\beta$-cell integrity. TQ was also found to successfully abrogate induction of diabetes mellitus with streptozotocin via nitric oxide inhibitory mechanism. ${ }^{6}$

Glycogen phosphorylase is one of the phosphorylase enzymes and it has been anticipated as one ways for curing diabetes-2. Since glucose production in the liver has been shown to increase in type 2 diabetes patients, inhibiting the release of glucose from the liver's glycogen's supplies appears to be a valid approach. ${ }^{6}$ The aim of this study was to elucidate the mechanisms underlying the glucose lowering effects of thymoquinone in vitro and in silico using glycogen phosphorylase enzyme assay and docking tools, respectively.

\section{MATERIALS AND METHODS}

\section{In vivo hypoglycemic properties of Thymoquinone}

Sprague Dawley rats (average body weight 150-200 g) were used in this study. Rats were housed in special clear sided cages at controlled temperature $\left(37^{\circ} \mathrm{C}\right)$ with a $12: 12$-h light: dark cycle and had free access to water and laboratory standard commercial food over a 2-week adaptation period. Rats were fasted for $12 \mathrm{~h}$ prior to induction of diabetes. Diabetes was induced in twenty five rats by a single intra-peritoneal injection of STZ (65 mg/kg b. w freshly dissolved in $5 \mathrm{mmol} / \mathrm{l}$ citrate buffer, $\mathrm{pH} 4.5$ ) 15 minutes after intra-peritoneal injection of Nicotinamide (NA) (120 $\mathrm{mg} / \mathrm{kg} \mathrm{b}$. w dissolved in normal saline). Rats were given $5 \%$ dextrose instead of water for the first 24 hours to avoid hypoglycemia. Diabetes 
was confirmed by blood glucose level of $\geq 200 \mathrm{mg} / \mathrm{dl}$. After confirmation of diabetes, diabetic rats were randomly divided into five groups. The $1^{\text {st }}$, $2^{\text {nd }}$ and $3^{\text {rd }}$ groups were treated with TQ at doses of 60,30 and $10 \mathrm{mg} /$ $\mathrm{kg}$, respectively, for sixty days. The $4^{\text {th }}$ group was treated with metformin at a dose of $140 \mathrm{mg} / \mathrm{kg}$ whilbetic control group. The sixth group served as non-diabetic normal control. At day zero fasting blood glucose was done and the animals were weighed. 4 weeks after commencement of the treatment, GTT before sacrificing animals. Any reduction in blood sugar level in comparison to that of untreated controls was taken as anti-diabetic activity. Ethical approval for this study was obtained from the Internal Board Review, Medical Research Centre, Jazan University.

\section{Glycogen phosphorylase enzyme assays}

\section{Materials}

Glycogen phosphorylase a (from rabbit muscle), glycogen, glucose-1-phosphate, malachite green, and ammonium molybdate were purchased from the Sigma-Aldrich Corporation. Reagents and solvents were obtained from commercial suppliers and used without further purification. Solvents used were AR grade.

\section{Enzymatic activity assay}

The enzymatic inhibition of phosphorylase activity was monitored using multiskan spectrum (Thermo-Scientific) based on the published methods. In brief, GPa activity was measured in the direction of glycogen synthesis by the release of phosphate from glucose-1-phosphate. $0.5 \mathrm{mM}$. Thymoquinone was dissolved in DMSO. The enzyme was added into the $100 \mu \mathrm{L}$ buffer with compounds dissolved in containing 50 $\mathrm{mM}$ Hepes ( $\mathrm{pH}$ 7.2), $100 \mathrm{mM} \mathrm{KCl}, 2.5 \mathrm{mM} \mathrm{MgCl}_{2}, 0.5 \mathrm{mM}$ glucose1-phosphate, and $1 \mathrm{mg} / \mathrm{mL}$ glycogen in 96-well microplates. After the addition of $150 \mu \mathrm{L}$ of $1 \mathrm{M} \mathrm{HCl}$ containing $10 \mathrm{mg} / \mathrm{mL}$ ammonium molybdate and $0.38 \mathrm{mg} / \mathrm{mL}$ malachite green, reactions were run at $25^{\circ} \mathrm{C}$ for $20 \mathrm{~min}$. And then the phosphate absorbance was measured at $620 \mathrm{~nm} .{ }^{9}$

\section{Docking with Auto-Dock 4.0 software}

Auto Dock Tools (ADT) is a program package of automated docking tools. It is designed to predict how small molecules, such as substrates or drug candidates, bind to a receptor of known 3D-structure. Besides generating binding energies in these docking studies, the position of the ligand in the receptor's binding site can be visualized. It can be useful for designing better ligands and understanding the nature of the binding. In this experiment you will model the binding of an inhibitor ligand to its target enzyme. Auto-Dock 4.0 search for the best ways to fit a ligand molecule into a receptor results in a docking log file that contains a detailed record of the docking. By convention, these results files have the extension (.dlg). Reading a docking log or a set of docking logs into Auto-Dock 4.0 is the first step in analyzing the results of docking experiments.

\section{Docking experiment}

Each docking requires at least four input files PDBQT file for the ligand that encodes a torsion tree, PDBQT file for the receptor, Grid parameter file (GPF) for the Auto Grid calculation and Docking parameter file (DPF) for the Auto Dock calculation. If some residues in the receptor are to be modeled as flexible, a fifth PDBQT file containing the flexible receptor residues will be necessary. Protein Data Bank (PDB) files may have a variety of problems that need to be corrected before they can be used in ADT. These potential problems include missing atoms, added waters, more than one molecule, chain breaks, alternate locations etc.

\section{Preparation of the ligand file}

The ligand originally is drawn in different visualization programs such as Chem3D Ultra to obtain standard 3D structures (.mol2) format. All hydrogen's was added to the ligands and gastiger partial atomic charges were computed and saved in the required format. Auto-Dock 4.0 automatically assigned type to each atom and detected root (this is the rigid part of the ligand) also choose torsions option was used as an interactive browser to choose the number of rotatable bonds that move the fewest atoms, finally the ligands was saved as pdbqt format.

\section{Preparation of the macromolecule file}

The receptor is usually taken from the Protein Data Bank (PDB) in (.pdb) format. This is a special format for protein structures that are obtained by X-ray crystallography studies. All polar hydrogens were added, and the partial kollman charge was computed for arbitrary molecule. The protein molecule was then saved as pdbq file. Protein was defined as two parts which are flexible residue (amino acids in the binding site region of the protein that are able to alter their position via conformation charge upon ligand binding) was selected to prepare flexible residue file, the second file was the rigid residue part.

\section{Preparation of the Grid parameter file}

Autogrid builds as many files as the number of probe atoms used. Each one shows the interaction energies for a particular atom type, such as aliphatic carbons, aromatic carbons, hydrogen bonding oxygens, and so on. The grid itself is a box with determined dimensions that is located in a place on the surface of the receptor where we expect the ligand to interact with the receptor. The center of such a grid was precalculated earlier and will be used in the following operations. An additional feature of Auto-Dock 4.0 is the ability to model not only how the ligand docks to the receptor but also the position of flexible residues. Flexible residues are amino acids that change their positions while the ligand interacts with the receptor. They are found by comparison of different crystallized structures or by molecular dynamic simulations. The grid volume should be large enough to at least allow the ligand to rotate freely, even when the ligand is in its most fully-extended conformation. To set up the grid size you can inspect it visually and adjust the dimension by using the three thumbwheel widgets. So for instance the suitable grid size was determined according to flexible residues, using the default grid spacing of 0.375 . In the case you do not have any ligand position information the grid should be centered in the putative binding site and sized to embrace all residues making the binding pocket. Adjust all the values and save the information, save the gpf file and check the written file.

\section{Preparation of the docking parameter file}

When the preparation of the input files (ligand and protein) and the calculation of the affinity maps are properly performed Auto-Dock will carry out the docking automatically using the newest docking algorithm (Lamarckian Genetic Algorithm). Prepared docking parameter file was the final step. The resultant structure files of Auto-Dock 4.0 software was analyzed using Discovery studio visualization (DSV) and ligplot programs.

\section{Statistical analysis}

Values are expressed as mean \pm SEM. Unpaired Student t-test was used for statistical comparison. In case of in vivo studies comparison were made between normal and diabetic, diabetic versus diabetic treated animals. Changes were considered significant if the P-value was less than 0.05 .

\section{RESULTS}

The current study employed a well established animal model of diabetes to investigate the hypoglycemic properties of TQ. Diabetes was induced using STZ and NA. As shown Table 1., the effect of this natural compound on the body weights and blood glucose levels. Diabetic rats treat- 
Table 1: Effects of Thymoquinone on body weight and fasting blood glucose of STZ induced type-2 rats

\begin{tabular}{|c|c|c|c|c|c|}
\hline \multirow{2}{*}{ Groups } & \multirow{2}{*}{ Initial weight (gm) } & \multirow{2}{*}{ Final weight (gm) } & \multicolumn{3}{|c|}{ Blood Glucose Levels (mg/dl) } \\
\hline & & & Fasting ( $0 \mathrm{hr})$ & $1 \mathrm{hr}$ & $2 \mathrm{hr}$ \\
\hline $\begin{array}{c}\text { STZ-induced }+ \\
\text { thymoquinone } 10 \mathrm{mg} / \mathrm{kg}\end{array}$ & $156.33 \pm 3.6$ & $135.83 \pm 2.9$ & $180.33 \pm 10.5$ & $346.5 \pm 5.1$ & $260.5 \pm 2.7$ \\
\hline $\begin{array}{l}\text { STZ-induced + } \\
\text { thymoquinone } 30 \mathrm{mg} / \mathrm{kg}\end{array}$ & $164.44 \pm 1.5$ & $140.97 \pm 6.4$ & $137.38 \pm 1.9$ & $285.58 \pm 2.9$ & $172.75 \pm 10.8$ \\
\hline $\begin{array}{c}\text { STZ-induced + } \\
\text { thymoquinone } 60 \mathrm{mg} / \mathrm{kg}\end{array}$ & $173.5 \pm 5.8$ & $307.83 \pm 8.4$ & $177 \pm 2.4$ & $306 \pm 5.7$ & $222 \pm 8.1$ \\
\hline $\begin{array}{l}\text { STZ-induced + } \\
\text { metformin }\end{array}$ & $179.6 \pm 6.4$ & $175.33 \pm 5.9$ & $102.6 \pm 65.1$ & $255 \pm 1.8$ & $87 \pm 5.8$ \\
\hline STZ-induced (Diabetic) & $155.25 \pm 2.9$ & $291.5 \pm 2.5$ & $257.5 \pm 5.7$ & $440 \pm 6.4$ & $326 \pm 6.4$ \\
\hline Normal group & $171.83 \pm 1.5$ & $184.16 \pm 3.5$ & $84.33 \pm 5.8$ & $122.16 \pm 3.8$ & $93.16 \pm 4.9$ \\
\hline \multicolumn{6}{|l|}{ ANOVA } \\
\hline F-statistic & 1.249 & 2.374 & 2.178 & 5.980 & 3.981 \\
\hline P-value & 0.322 & 0.068 & 0.04 & 0.002 & 0.011 \\
\hline
\end{tabular}

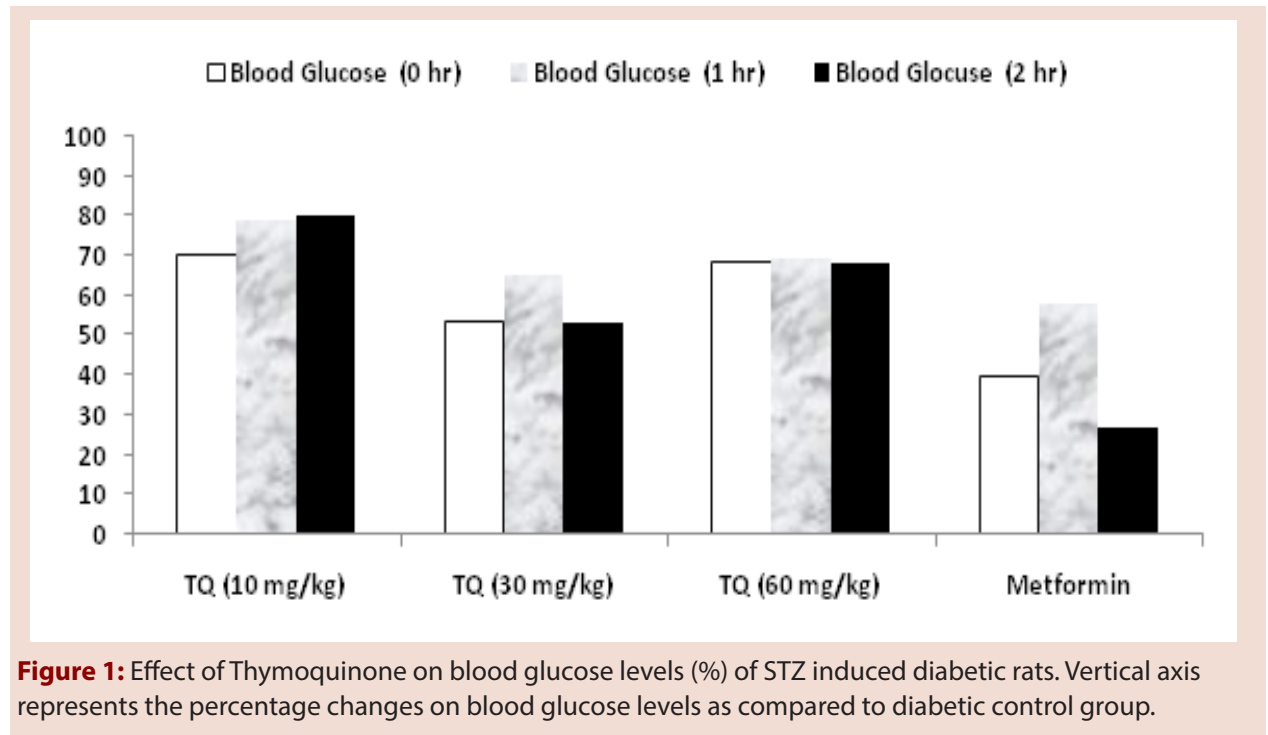

ed with TQ oral dose of 10, 20 and $30 \mathrm{mg} / \mathrm{kg}$ showed $70.03 \%, 53.35 \%$, $68.74 \%$, respectively, reduction in blood glucose (at zero time) in comparison to untreated diabetic rats. Figure 1 showed the effect of TQ on blood glucose levels (\%) of STZ-NA induced diabetic rats. Vertical axis represents the percentage changes on blood glucose levels as compared to diabetic control group. Blood glucose levels of control diabetic and treated animals were $102.6 \pm 65.1$ and $257.5 \pm 5.7 \mathrm{mg} / \mathrm{dl}$ respectively $(P<0.05)$. Changes in body weight are depicted in Table 1 .

As shown in Table 2, TQ at a concentration of $0.05 \mathrm{Mm}$ inhibits GPa activity by only $14.9 \%$. To investigate the interaction of TQ, molecular docking simulations of the binding of this molecule with glycogen phosphorylase active site were carried out using Autodock 4.2. Biochemical interactions of TQ with GPa is shown in Figure 2. Figure 2a is schematic diagram obtained by DSV software for docking results of Auto-Dock while Figure $2 \mathrm{~b}$ is a schematic diagram obtained by Ligplot software for docking results of Auto-Dock. Caffeine was used as standard in glycogen phosphorylase assay.

\section{DISCUSSION}

The antidiabetic property of N. sativa seeds oil is attributable to the presence of thymoquinone (TQ) $)^{10,11}$ On the other hand many studies have been designed to investigate the possible effects of the TQ in STZ-NA
Table 2: Effects of Thymoquinone on Glycogen phosphorylase enzyme (Protein code: 1GFZ from protein data bank)

\begin{tabular}{ccc}
\hline Compound & $\begin{array}{c}\text { Inhibition \% of G Pase } \\
(0.5 \mathrm{Mm})\end{array}$ & Docked Energy \\
\hline Thymoquinone & 14.9 & -5.58 \\
\hline
\end{tabular}

induced diabetes in rats. The aim of this study was to elucidate the mechanisms underlying the glucose lowering effects of Thymoquinone in vitro and in silico using glycogen phosphorylase (GPa) enzyme assay and docking tools, respectively.

For the study of antidiabetic agents, STZ induced hyperglycemia in rodents is considered to be a good preliminary screening model and is widely used. ${ }^{12,13} \mathrm{STZ}, \mathrm{N}$-[methylnitrocarbamoyl]-D-glucosamine is a potent methylating agent for DNA and acts as nitric oxide donor in pancreatic cells. Beta cells are particularly sensitive to damage by nitric oxide and free radicals because of their low levels of free radical scavenging enzymes. ${ }^{14-17}$ From the results obtained, it is evident diabetic rats had much higher glucose levels than of control rats. Oral administration of TQ decreased the blood glucose level in diabetic rats. Anti-hyperglycemic effect of medicinal plant extracts is generally dependent upon the degree of $\beta$-cell destruction. ${ }^{18,19}$ 


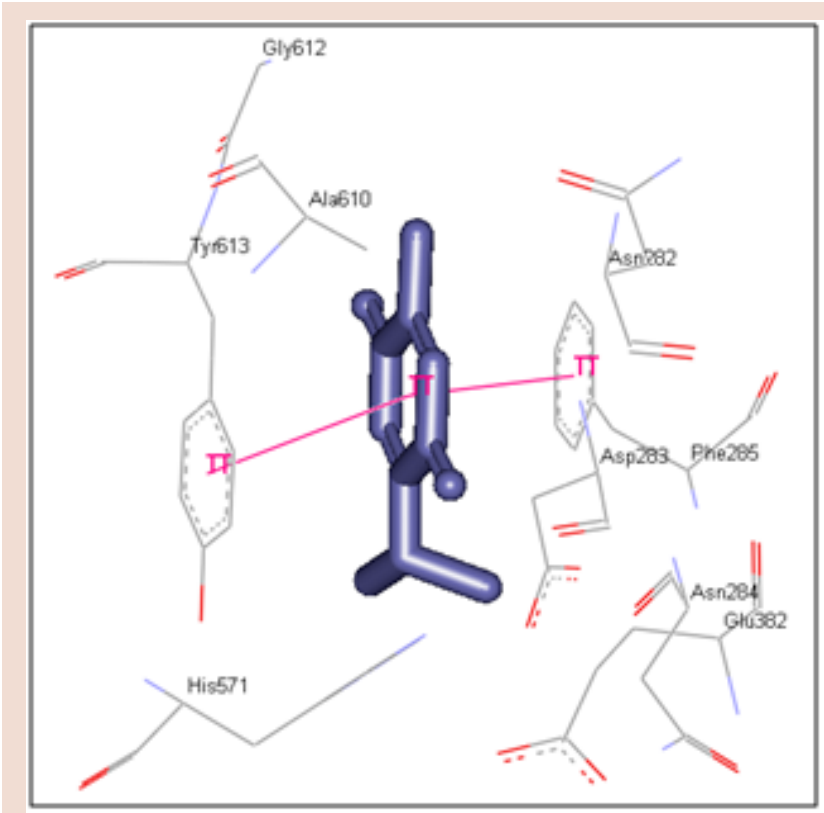

a

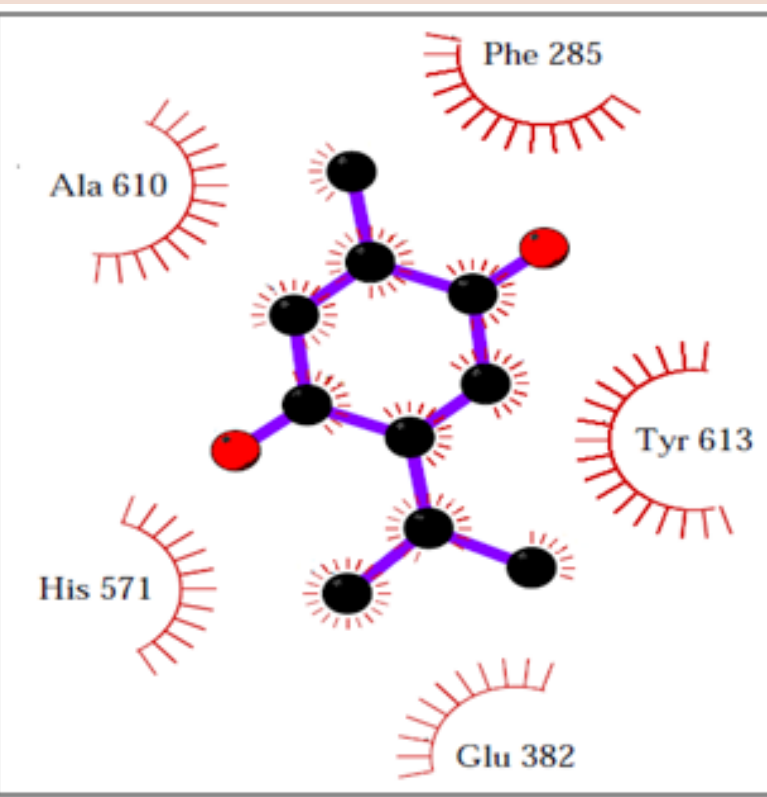

$\mathrm{b}$

Figure 2: Biochemical interactions of Thymoquinone with Glycogen phosphorylase: (A) is schematic diagram obtained by DSV software for docking results of Auto-Dock. (B) is schematic diagram obtained by Ligplot software for docking results of Auto-Dock. Protein code: 1 GFZ from protein data bank.

In spite of the availability of different classes of hypoglycaemic drugs, treatment regimens are often unable to achieve an intensive degree of glucose control known to most effectively reduce the incidence and severity of diabetic complications. ${ }^{20,21}$ Hepatic glucose output is elevated in type 2 diabetic patients and current evidence indicates that glycogenolysis (release of monomeric glucose from the glycogen polymer storage form) is an important contributor to the abnormally high production of glucose by the liver. ${ }^{22,23}$ Glycogen phosphorylase is the enzyme that catalyses this release and recent advances in new inhibitors of this structurally and kinetically well studied enzyme have enabled work which further delineate the pharmacological and physiological consequences of inhibiting glucose production by this pathway. ${ }^{24,25}$ Most notably, these agents lower glucose in diabetic animal models, both acutely and chronically, appear to affect both gluconeogenic and glycogenolytic pathways and demonstrate potential for a beneficial effect on cardiovascular risk factors. Results of the current study did not show any significant inhibition of glycogen phosphorylase by TQ. As shown in Table 2, TQ at a concentration of $0.05 \mathrm{Mm}$ inhibits GPa activity by only $14.9 \%$. To investigate the interaction of TQ, molecular docking simulations of the binding of this molecule with glycogen phosphorylase active site were carried out using Autodock 4.2.

More studies are needed to demonstrate the exact mechanism of action of TQ on diabetes. Consequently, TQ may be clinically useful for the treatment of diabetes.

\section{CONCLUSION}

The current results show that TQ is associated with potential antihyperglycemic effects. Furthermore, anti-diabetic properties of TQ are unassociated with glycogen phosphorylase inhibition.

\section{ACKNOWLEDGMENT}

This research was graciously supported the Deanship of Scientific Research, Jazan University (35/5/45) and the Scientific Chair for Prophetic Medicine and Scientific Miracle.

\section{CONFLICT OF INTEREST}

The authors report no conflicts of interest in this work.

\section{REFERENCES}

1. Burits M, Bucar F. Antioxidant activity of Nigella sativa essential oil. Phytotherapy Research 2000; 14(5): 323-8.

2. Ali BH, Blunden G. Pharmacological and toxicological properties of Nigella sativa. Phytotherapy Research 2003; 17(4): 299-305.

3. Mariod AA, Ibrahim RM, Ismail M, Ismail N. Antioxidant activity and phenolic content of phenolic rich fractions obtained from black cumin (Nigella sativa) seedcake. Food Chem. 2009; 116(6): 306-12.

4. Bourgou S, Pichette A, Marzouk B, Legault J. Bioactivities of black cumin essential oil and its main terpenes from Tunisia. South African Journal of Botany 2010; 76(6): 210-6.

5. El-Abhar HS, Abdallah DM, Saleh S. Gastroprotective activity of Nigella sativa oil and its constituent, thymoquinone, against gastric mucosal injury induced by ischaemia/reperfusion in rats. Journal of ethnopharmacology 2003; 84(2): 251.

6. Ravindran J, Nair HB, Sung B, Prasad S, Tekmal RR, Aggarwal BB. Thymoquinone poly (lactide-co-glycolide) nanoparticles exhibit enhanced anti-proliferative, antiinflammatory, and chemosensitization potential. Biochemical pharmacology 2010; 79(11): 1640-17.

7. Zaoui A, Cherrah Y, Lacaille-Dubois MA, Settaf A, Amarouch H, Hassar M. Diuretic and hypotensive effects of Nigella sativa in the spontaneously hypertensive rat. Therapie 2000; 55(3): 379-401.

8. Salem ML. Immunomodulatory and therapeutic properties of the Nigella sativa L. seed. International immunopharmacology 2005; 5(13): 1749-70.

9. Martin WH, Hoover DJ, Armento SJ, et al. Discovery of a human liver glycogen phosphorylase inhibitor that lowers blood glucose in vivo. Proc Natl Acad Sci U S A. 1998; 95(4): 1776-81.

10. Hamdy NM, Taha RA. Effects of Nigella sativa oil and thymoquinone on oxidative stress and neuropathy in streptozotocin-induced diabetic rats. Pharmacology 2008; 84(3): 127-34.

11. Pari L, Sankaranarayanan C. Beneficial effects of thymoquinone on hepatic key enzymes in streptozotocin-nicotinamide induced diabetic rats. Life Sciences 2009; 85(23): 830-4

12. Szkudelski T. The mechanism of alloxan and streptozotocin action in B cells of the rat pancreas. Physiological research 2001; 50(06): 537-46.

13. Yamamoto $H$, Uchigata $Y$, Okamoto $H$. Streptozotocin and alloxan induce DNA strand breaks and poly (ADP-ribose) synthetase in pancreatic islets; 1981. 284-6.

14. Rao NK, Nammi S. Antidiabetic and renoprotective effects of the chloroform extract of Terminalia chebula Retz. seeds in streptozotocin-induced diabetic 
rats. BMC complementary and alternative medicine 2006; 6(1): 17.

15. Singh SN, Vats $P$, Suri $S$, et al. Effect of an antidiabetic extract of Catharanthus roseus on enzymic activities in streptozotocin induced diabetic rats. Journal of Ethnopharmacology 2001; 76(3): 269-77.

16. Latha M, Pari L. Preventive effects of Cassia auriculata L. flowers on brain lipid peroxidation in rats treated with streptozotocin. Molecular and cellular biochemistry 2003; 243(1-2): 23-8.

17. Latha M, Pari L, Sitasawad S, Bhonde R. Insulin-secretagogue activity and cytoprotective role of the traditional antidiabetic plant Scoparia dulcis (Sweet Broomweed). Life sciences 2004; 75(16): 2003-14.

18. Grover J, Yadav S, Vats V. Medicinal plants of India with anti-diabetic potential. Journal of ethnopharmacology 2002; 81(1): 81-100.

19. Maiti R, Jana D, Das U, Ghosh D. Antidiabetic effect of aqueous extract of seed of Tamarindus indica in streptozotocin-induced diabetic rats. Journal of Ethnopharmacology 2004; 92(1): 85-91.

20. Treadway JL, Mendys P, Hoover DJ. Glycogen phosphorylase inhibitors for treatment of type 2 diabetes mellitus. Expert opinion on investigational drugs 2001; 10(3): 439-54.

21. Kristiansen $M$, Andersen $B$, Iversen LF, Westergaard N. Identification, synthesis, and characterization of new glycogen phosphorylase inhibitors binding to the alIosteric AMP site. Journal of medicinal chemistry 2004; 47(14): 3537-45.

22. Nagy V, Felföldi N, Kónya B, et al. N-(4-Substituted-benzoyl)-N'-( $\beta$-d glucopyranosyl) ureas as inhibitors of glycogen phosphorylase: synthesis and evaluation by kinetic crystallographic, and molecular modelling methods. Bioorganic and medicinal chemistry 2012; 20(5): 1801-16.

23. Nagy V, Benltifa M, Vidal S, et al. Glucose-based spiro-heterocycles as potent inhibitors of glycogen phosphorylase. Bioorganic and medicinal chemistry 2009; 17(15): 5696-707.

24. Moller DE. New drug targets for type 2 diabetes and the metabolic syndrome. Nature 2001; 414(6865): 821-7.

25. Rath VL, Ammirati M, Danley DE, et al. Human liver glycogen phosphorylase inhibitors bind at a new allosteric site. Chemistry and biology 2000; 7(9): 677-82.

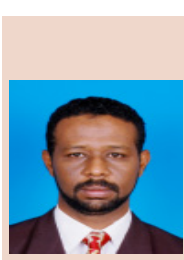

\section{ABOUT AUTHORS}

Dr. Siddig Ibrahim Abdelwahab: Is Associate professor in Jazan University, Jazan, KSA. He graduated from Faculty of Veterinary SciencesUniversity of Khartoum in the field of Veterinary Medicine in the year 1999 and obtained his Master of Science and Doctor of Philosophy Degrees in Pharmacology from University Putra Malaysia, Malaysia in the years 2001 and 2008 respectively. He also had opportunity to join as Head of the Biomedical Research Unit, (Medical Research Centre, and Substance Abuse Research Centre) and Core Laboratory, Medical Research Centre, Jazan, Saudi Arabia. In his attempt to share his expertise, he acquired an overall H-index of 19 with his high number of research publications (130) and two patents filed. He is active member of four scientific communities and is been considered as potential reviewer for more than 10 biomedical journals. His excellence in research also extends to statistical analysis. 